\title{
TWIST PERIODIC ORBITS FOR CONTINUOUS MAPS OF THE EIGHT SPACE
}

\author{
Associate professor Iftichar Mudhar Talb Al-shraa \\ Iraq, Babylon University, \\ College of Education for Pure Science
}

Keywords: Twist map, Eight space, Rotation set, Periodic set.

Abstract. Let $g$ be a continuous map from 8 to itself has a fixed point at $(0,0)$,we prove that $g$ has a twist periodic orbit if there is a rational rotation number

\subsection{Introduction:}

In [1], Alseda,L. et.al introduced the definition of twist periodic orbit, they studied this type of periodic on continuous circle map of degree one which has a fixed point In.[4], ] Misurewicz,M studied the twist sets for circle maps. Let 8 be the one point union of two unit circles attached at $(0,0)$ and $, g: 8 \rightarrow 8$ be a map has a fixed point at $(0,0)$, we say that $z \in 8$ is a periodic point of $g$ if there exists a positive integer $\mathrm{n}$ such that $g^{n}(z)=z$. The period of $\mathrm{z}$ is the smallest integer satisfying this relation. Let $p(g)$ be the set of periods of $g$. if $z \in 8$ is a periodic point of period $n$, then the orbit of $\mathrm{z}$ is the set $\left\{g^{i}(z): i=1,2, \ldots, n\right\}$ Throughout this work, we will use e as projection from $s^{1}$ onto 8 . There exists many projections from $s^{1}$ into 8 , but we define e as: $e(z)= \begin{cases}i\left(1-z^{2}\right) & , \quad \forall z \in s^{1} i m(z) \geq 0 \\ i\left(z^{2}-1\right) & \forall z \in s^{1} i m(z)<0\end{cases}$

This projection is one to one. We can find a lift map f from $s^{l}$ to itself such that $e g=f e$ Let $\mathrm{G}$ be a lift to $g$ and $e \pi(t)=e(\cos 2 \pi t+i \sin 2 \pi t)$ a projection of $\mathrm{R} \rightarrow 8$. It is clear $\pi$ is many to one, $G$ is not defined uniquely; that is if $G_{1}$ and $G$ are two lifting of $g$ then $\mathrm{G}=G_{1}+m$ with $m \in Z$. the degree of $g$ is the number $a$ which satisfy $G(t+1)=G(t)+\alpha, \forall t \in R$. In this work, we use $a=1$. If $z \in 8 \mathrm{~s}$ a periodic point of $g$ of period $\mathrm{n}$ and $e \pi(t)=z$ then $G^{n}(t)=t+k$; where $k \in Z$ this imply $\frac{k}{n}$ is the rotation number of z we denoted it by $\rho(G, z)$. We denote by $L_{G}(g)$ the set of all rotation numbers of $g$, so $L_{G}(g)=\{\rho(G, z): z \in 8 ; G: R \rightarrow R\}$ is called the rotation set of $g$. Since $f$ is unique lift to $g$ then the properties of rotation number and rotation set on the circle satisfy on the eight space. So the rotation set does not depend on the choice of $t$, but it depends on the periodic orbit. Also, if $\left(a_{i}\right)$ is a convergent sequence of a and $a_{i} \in L_{G}(g)$ then $a \in L_{G}(g)$.' The another property $\rho(G, z)=\rho\left(G_{1}, z\right)+k$ if $G=G_{1}+k$. If $g$ has no periodic point the rotation number exist for all $t \in R$ and it is independent of $t$ and is irrational number there are more properties on circle maps satisfy on 8 (see [2] and [3]) .Let $z_{1}, z_{2} \in 8$ we denote $\left(z_{1}, z_{2}\right)$ (resp. $\left.\left[z_{1}, z_{2}\right]\right)$ the open (resp. closed) arc of 8 from $z_{1}$ counter clockwise to $z_{2}$ Let $\frac{d}{m}$ be a rational number, we assume $\mathrm{m}>0$ and let $\mathrm{T}$ be a periodic orbit of period $m$ and rotation number $\frac{d}{m}$ with $(d, m)=1$. Let $G$ be lift such that $G$ is order preserving on the set $(e \pi)^{-1} T$ then we say that $\mathrm{T}$ is a twist periodic orbit (for simplify we say TPO) of $g$ period $\mathrm{m}$ and rotation number $\frac{d}{m}$ It is clear, every periodic orbit of period 1 is a TPO. If $\frac{d}{m}>0$ then $\left(z_{i}, z_{i+1}\right) \cap T=\emptyset, \forall i=1,2, \ldots, m-1$ and $\left(z_{m}, z_{1}\right) \cap T=\emptyset$, and if $\frac{d}{m}<0$ 
$\left(z_{i+1}, z_{i}\right) \cap=\emptyset, \forall i=1,2, \ldots, m-1$ and $\left(z_{1}, z_{m}\right) \cap T=\emptyset$. Then notion of TPO of period $\mathrm{m}$ characterizes the simplest behavior of the graph a map which has the same rotation number.

\subsection{Main Theorems}

In this section, we prove two theorems which find the TPO, also we prove other properties of it.

Proposition 1.2.1: If $<\frac{k}{n}<c ; a, c \in L_{G}(g)$ and $k, n \in Z$ with $n>0$ and $\frac{k}{n} \in L_{G}(g)$ then $n \in p(g)$.

Proof: Suppose $\rho(G, z)=\frac{k}{n}$ thus there exists $\mathrm{z} \in 8$ such that there is $\mathrm{t} \in \mathrm{R}$ and $\mathrm{e} \pi(\mathrm{t})=\mathrm{z}$ then by definition $\rho(G, z)=\lim _{n \rightarrow \infty} \sup \frac{G^{n}(t)-t}{n}=\frac{k}{n} \quad$ so there is $\epsilon>0$ such that $-\epsilon<\frac{G^{n}(t)-t}{n}-\frac{k}{n}<\epsilon$ then $\frac{k}{n}-\epsilon<\frac{G^{n}(t)-t}{n}<\frac{k}{n}+\epsilon \quad$ Hence $\quad \frac{k+t}{n}-\epsilon<\frac{G^{n}(t)}{n}<\frac{k+t}{n}+\epsilon$,

hus

$(k+t)-n \epsilon<G^{n}(t)<(k+t)+n \epsilon$ Since $\epsilon$ is an arbitrary and $G^{n}(t) \in R$ is any point then $G^{n}(t)=t+k$ that is $\mathrm{t}$ is a periodic point of $\mathrm{G}$ of period $\mathrm{n}$ then by properties of the lift then $\mathrm{g}$ has periodic of period $\mathrm{n}$ then $n \in p(g)$

Corollary 1.2.2: $L_{G}(g) \cap \frac{z}{2} \neq \emptyset$ if and only if $z=0$ is the only fixed point of $g$.

Proof: If $\mathrm{z}=0$ then there exists $t \in \frac{z}{2}$ such that $e \pi(t)=z=0$.Let $L_{G}(g) \cap \frac{z}{2} \neq \emptyset$ so there is $\frac{k}{2} \in L_{G}(g) \cap \frac{z}{2}$ hence there is $z \in 8$ such that $\rho(G, z)=\frac{k}{2}$ so there is $t \in Z, e \pi(t)=0$.

Corollary 1.2.3: $L_{G}(g) \cap Z \neq \emptyset$ if and only of $\mathrm{g}$ has a fixed point $z \neq 0$

Proof $: \rightarrow$ Since $L_{G}(g) \cap Z \neq \emptyset$ then there is an integer number $k \in L_{G}(g)$,so by proposition 1.2.1, $1 \in p(g)$ In other word $g$ has a fixed point

$\leftarrow)$ since $1 \in p(g)$ then there $z \in 8$ such that $g(z)=z$, so $G(t)=t+k$; where

$k \in Z$, thus $\rho(G, z)=\frac{k}{1} \in L_{G}(g)$ then $k \in L_{G}(g) \cap Z \neq \emptyset$.

The proposition below gives a geometrical interpretation of a TPO on the eight space :

Proposition 1.2.4: Let $g: 8 \rightarrow 8$ be a continuous map, $T=\left\{z_{1}, z_{2}, \ldots, z_{m}\right\}$ be a TPO of period $\mathrm{m}$ and rotation number $\frac{d}{m}$ Then $g\left(z_{i}\right)=z_{i+|d| \bmod m}$ if $z_{i} \neq 0$ where $\mathrm{i}=1,2, \ldots, \mathrm{m}$.

Proof: Suppose $\frac{d}{m}>0$ let $\mathrm{G}$ be the lifting of g for which $\rho\left(G, z_{i}\right)=\frac{d}{m},(e \pi)^{-1} T=\left\{t_{i}: i \in Z\right\}$ with $\ldots<t_{-2}<t_{-1}<t_{0}<t_{1}<t_{2}<\cdots$. Assume $e \pi\left(t_{1}\right)=z_{1}$. We claim $t_{i+r m}=t_{i}+r$, if $t_{i} \in \frac{z}{2}$. Since $t_{i}$ is a periodic point of period $\mathrm{m}$. then $e \pi\left(t_{i+r m}\right)=e \pi\left(t_{i}\right) \cdot$ 'Thus $\pi\left(t_{i+r m}\right)=\pi\left(t_{i}\right) ; t_{i} \in \frac{z}{2}$, so $t_{i+r m}=t_{i}+r$. Also, we claim $t_{i+r m}=t_{i}+\frac{r}{2}$, if $t_{i} \in \frac{z}{2}$. Then $\left(t_{i+r m}\right)=e\left(-\pi\left(t_{i}\right)\right)=-e \pi\left(t_{i}\right)$ so $\quad$ then $\pi\left(t_{i+r m}\right)=-\pi\left(t_{i}\right)$.Then $t_{i+r m}=t_{i}+\frac{r}{2} . \quad$ Case $\quad 1$ : $e \pi\left(t_{i+r m}\right)=e \pi\left(t_{i}\right)=e \pi\left(t_{i}\right)=z_{i}$, for $k \in Z$ and $i=1,2, \ldots, m$, since $\mathrm{F}$ on $(e \pi)^{-1} T_{\text {is one }}$ to one and by definition of TPO $(e \pi)^{-1} T$ order preserving then $G\left(t_{i}\right)=t_{i+j}$; for some $j \in Z$ and all $i$,so $G^{2}\left(t_{i}\right)=G\left(G\left(t_{i}\right)\right)=G\left(t_{i+j}\right)=t_{i+2 j}$ thus by induction we get $G^{m}\left(t_{i}\right)=t_{i+m j}$ Since $t_{i}$ is a periodic 
point of period $\mathrm{m}$ and $\rho(G, z)=\frac{d}{m}$ then $t_{i}+d=G^{m}\left(t_{i}\right)$ $=G^{m-1}\left(G\left(t_{i}\right)\right)=G^{m-1}\left(t_{i+j}\right)=\ldots=t_{i+m j}=t_{i}+j \quad$ (by claim $\quad 1$ ) then $d=j$ hence $g\left(z_{i}\right)=Z_{i+d(\bmod m)} ; \forall i=1,2, \ldots, m$. Since Tis an orbit not a union of several orbits then $(d, m)=1$ Case 2: If $t_{i} \in \frac{z}{2} \quad, e \pi\left(t_{i+r m}\right)=e \pi\left(t_{i}+\frac{r}{2}\right)=e\left(-\pi\left(t_{i}\right)\right)=-e \pi\left(t_{i}\right)=0$. Since, F on $(e \pi)^{-1} T$ is one to one and by definition of TPO $(e \pi)^{-1} T$ order preserving then $G\left(t_{i}\right)=t_{i+j}$; for some $\mathrm{j} \in \frac{z}{2}$ in the same way $t_{i}+d=G^{m}\left(t_{i}\right)=G^{m-1}\left(t_{i+j}\right)=\ldots=t_{i+m j}=t_{i}+j$ (by claim 2)thus $d=j$; where $\mathrm{j} \in \frac{z}{2}$ this imply $g\left(z_{i}\right)=f(0)=0$, so $z_{i}$ is an eventually fixed point.

An A-graph of $\mathrm{g}$ with respect to $\mathrm{A}_{m}$ and $\mathrm{B}_{m}$ is an oriented graph with vertices $I_{1}, I_{2}, \ldots, I_{m+n-1}$ such that if $I_{a} f$ covers $I_{\beta} \mathrm{k}$ times but not $\mathrm{k}+1$ times then there are $\mathrm{k}$ arrows from $I_{a}$ to $I_{\beta}$. a sequence $I_{i_{0}} \rightarrow I_{i_{1}} \rightarrow \cdots \rightarrow I_{i_{r}}$ is an A-graph of $\mathrm{g}$ is called a path of length $\mathrm{r}$ and the path is called a loop if $I_{i_{\mathrm{o}}}=I_{i_{r}}$ a loop is called simple if $I_{i_{j}} \neq I_{i_{k}}$ for $0 \leq j<k<r$.

The lemma below generalize lemma in[2] on circle map:

Lemma 1.2.5: Let $g: 8 \rightarrow 8$ be a continuous map of degree one. If $J_{0} \rightarrow J_{1} \rightarrow \cdots \rightarrow J_{n-1} \rightarrow J_{0}$ is a loop in a A-graph of $\mathrm{g}$ then there exists a fixed point $\mathrm{z}$ of $\mathrm{g}^{\mathrm{n}}$ such that $g^{i}(z) \in J_{i}$ for $i=1,2, \ldots, n-1$. Lemma 1.2.6: Let $g$ be an monotone map (not necessarily continuous) of a closed interval I into itself. Then $g$ has a fixed point.

Proof: suppose $\mathrm{g}$ is increasing map Let $\mathrm{t}=\sup \{y: g(y)>y\} \neq 0$ since $g$ is increasing, if $t<g(t)$ then $\forall y \in(t, g(t))$ then $g(y)>g(t)>y$ but this contraction.

If $t>g(t)$ then $\forall y \in(t, g(t))$ then $g(y)<g(t)<y$ but this contraction. Thus $\mathrm{t}=\mathrm{g}(\mathrm{t})$ that is, $\mathrm{t}$ is a fixed point. If $\mathrm{g}$ is decreasing map then the proof is similar

Let $A=\left\{a_{1}, a_{2}, \ldots, a_{n}\right\}$ be an invariant set that is $g(A) \subset A$, let $t_{1}<t_{2}<\cdots<t_{n}<t_{n}+1$ be such that $e \pi\left(t_{i}\right)=a_{i}$ for $\mathrm{i}=1,2, \ldots, \mathrm{n}$. Let $\mathrm{G}$ be a lift of $\mathrm{g}$, we denote by $\bar{G}$ the map such that :

1) $\bar{G}\left(t_{i}+k\right)=G\left(t_{i}+k\right)$ for $\mathrm{i}=\mathrm{I}, 2, \ldots, \mathrm{n}$ and $k \in Z$.

2) $\bar{G}$ on $\left[t_{i}+k, t_{i-1}+\mathrm{k}\right]$ is linear for $\mathrm{i}=1,2, \ldots, \mathrm{n}$ and $k \in Z$.

3) $\bar{G}$ on $\left[t_{n}+k, t_{n-1}+\mathrm{k}\right]$ is linear for $k \in Z$.

We call $\bar{G}$ the A linearization of G. we denote by $\bar{g}$ the map of 8 of degree one which has $\bar{G}$ as lift, also we say $\bar{g}$ the A linearization of $g$. If $\bar{G}=g$ that is $\bar{g}=g$ then we say that $\mathrm{G}$ and $g$ are linear.

Lemma 1.2.7: Let $g: 8 \rightarrow 8$ be a continuous map and A be an invariant subset under $\mathrm{g}$. let $\bar{g}$ be the A linearization of $\mathrm{g}$ and suppose that $\bar{g}$ has a TPO T of period $\mathrm{s}$ and $\rho(\bar{G}, z)=\frac{r}{s}$ with $(\mathrm{r}, \mathrm{s})=1 ;$ [ where $\bar{G}$ is the lift of $\bar{g}$ obtained by A linearization of G. If $T \not \subset A$ and $\bar{G}$ is increasing at every point of $(e \pi)^{-1} T$ then $\mathrm{g}$ has a TPO of period s and $\rho(G, z)=\frac{r}{s}$.

Proof: Let $t_{1}<t_{2}<\cdots<t_{n}<t_{1}+1$ such that $e \pi\left\{t_{1}, t_{2}, \ldots, t_{n}\right\}=A$ and $I_{i}=\left[t_{i}, t_{i+1}\right], i=1,2, \ldots, n-1$ and $I_{n}=\left[t_{n}, t_{1}+1\right]$ be subsets of R. Let $\mathrm{U}$ be the partition of $\mathrm{R}$ given by the points of $(e \pi)^{-1} A$, since $T \not \subset A ; \mathrm{T}$ is a periodic orbit and $\mathrm{A}$ is invariant set for $\bar{g}$ so $\bar{g}(A) \subset A$ then $\mathrm{T}$ and A are disjoint that is $T \cap A=\emptyset$. We choose $z \in T$ this 
imply there is unique $i_{1} \in\{1,2, \ldots, n\}$ such that there is $t \in I_{i_{1}}$ with $e \pi(t)=Z$ hence there is unique $i_{1} \in\{1,2, \ldots, n\}$ and $n_{j} \in Z$ such that $\bar{G}^{j-1}(t) \in t_{i_{j}}+n_{j}, j=1,2, \ldots, s+1$ since $(\bar{G}, z)=\frac{r}{s} ; \bar{g}_{\text {has a TPO pf period s. Then }} \bar{G}^{s}(t)=t+r$ thus $i_{s+1}=i_{1}$ and $n_{s+1}=r$ In such a way we obtain the following path in the U-graph of $\bar{G}: I_{i_{1}} \rightarrow I_{i_{2}}+n_{2} \rightarrow \cdots \rightarrow I_{i_{s}}+n_{s} \rightarrow I_{i_{1}}+r$ We will define $M_{s}: I_{i_{1}}+r \rightarrow I_{i_{s}}+n_{s}$ and $M_{j}: I_{i_{j+1}}+n_{j+1} \rightarrow I_{i_{j}}+n_{j} \quad \mathrm{j}=1,2, \ldots, \mathrm{s}-1$ such that $M_{j}(t)=\inf \left\{y \in I_{i_{j}}+n_{j}: G(y)=t\right\}$. Since $\bar{G}_{\text {is increasing on every interval }} I_{i_{j}}+n_{j}, j=1,2, \ldots, s$ these maps $M_{j}, M_{s}$ are increasing. Therefore $M: I_{i_{1}}+r \rightarrow I_{i_{1}}+r$ such that $=M_{1} \circ M_{2} \circ \ldots \circ M_{s}+r_{\mathrm{t}}$ is clear $\mathrm{M}$ is increasing. By lemma 1.2.6, $\mathrm{M}$ has a fixed point $p \in I_{i_{1}}+r_{\text {let }} p_{1}=p-r \in I_{i_{1}}$ thus $G^{j}\left(p_{1}\right) \in I_{i_{j+1}}+n_{j+1} ;$ for $j=0,1, \ldots, s-1$ and $G^{s}\left(p_{1}\right)=p_{1}+r \in I_{i_{1}}+r$ then the orbit $X=\left\{e \pi\left(p_{1}\right), g\left(e \pi\left(p_{1}\right)\right), \ldots, g^{s-1}\left(e \pi\left(p_{1}\right)\right)\right\}$ $\rho\left(G, e \pi\left(p_{1}\right)\right)=\frac{r}{s}$ We claim $\mathrm{X}$ is a TPO, to show that :Since $T=\left\{z_{1}, z_{2}, \ldots, z_{s}\right\}$ periodic orbit on $(e \pi)^{-1} T \subset R$ that is $\left\{G^{j}\left(p_{1}\right): j=0,1, \ldots, s-1\right\}+Z \subset R$. Now we will compare between

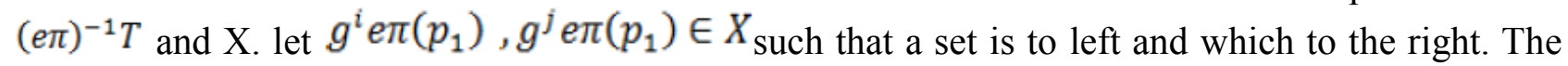
elements of the U-partition are :Case 1: If these intervals are different then $X=\left\{g^{i}\left(e \pi\left(p_{1}\right)\right): i=0,1, \ldots, s-1\right\}$ are the end points of U-partition, therefore we get $\mathrm{X}$ is a TPO. Case 2: If they are the same, then one has to go along the orbits of these two points, since we only use increasing pieces of the maps $G$ and $\bar{G}$ then $\left\{G^{j}: j=0,1, \ldots, s-1\right\}+Z$ and $(e \pi)^{-1} T$ in the same order, since T is a TPO so $\mathrm{X}$ is a TPO.

We will define these maps as: let $a, b \in R$ and $\mathrm{G}$ be a lift of $g G_{r}(a)=\sup \{G(b): b \leq a\}$ and $G_{l}(a)=\inf \{G(b): b \geq a\}$. We call $g_{r}: 8 \rightarrow 8$ such that $G_{r}$ is a lift of $g_{r}$ and $g_{r}: 8 \rightarrow 8$ such that $G_{l}$ is a lift of $g_{l}$ this mean $g_{r}, g_{l}$ are continuous maps of degree one.

Lemma 1.2.8: Let $g: 8 \rightarrow 8$ be a continuous map of degree one and $W$ be a periodic orbit of $g$ of period $\mathrm{m}$ and $\rho(G, z)=\frac{d}{m}$ such that $0<\frac{d}{m}<1$ and $(d, m)=1$. suppose that $W$ is not a TPO. then

$\mathrm{i}-g$ has a TPO R of period $\mathrm{s}$ and rotation number $\frac{r}{s}$ with $(r, s)=1$ and $\frac{d}{m}<\frac{r}{s}$. ii- $g$ has a TPO L of period $\mathrm{n}$ and rotation number $\frac{j}{n}$ with $(j, n)=1$ and $\frac{j}{n}<\frac{d}{m}$.

Proof : Without loss of generality,If we assume $g$ is linear on $\mathrm{W}$ and $\mathrm{F}$ is increasing on $(e \pi)^{-1} R$ then this don't for generality.Then by lemma 1.2.7, let $\mathrm{w}$ be a periodic orbit of $g$ of period $\mathrm{m}$ and $(G, z)=\frac{d}{m}$. let $\mathrm{U}$ be the partition of 8 by elements of $\mathrm{W}$, since $g$ is onto then for all $I \in U \exists J \in U$. Such that $g_{r}$ - covers $I$ since the number of intervals of $U$ is finite so we have a loop of length $\mathrm{s}$ in the U-graph of $g_{r}$ with $1 \leq s \leq m$. Assume that the loop is the shortest one of the U-graph of $g_{r}$ ince $\mathrm{W}$ is not a TPO at least on interval $I \in U$ satisfy $\left(g_{r} \mid I\right)=$ constant then $s<m$. By lemma 1.2.5, this loop gives us a periodic orbit $\mathrm{R}$ of $g_{r}$ of period s and rotation number $\stackrel{r}{s}$. since $s<m$ then $R \neq W$ all intervals on which $G_{r}$ is non decreasing (constant or increasing) then $\mathrm{R}$ is a TPO for $g_{r}$ this imply $\mathrm{R}$ is a TPO for $\mathrm{f}$. By proposition 1.2.4, we get $(\mathrm{r}, \mathrm{s})=1$. Now, we need prove that 
$\frac{r}{s}<\frac{d}{m}$. Since $G_{r}$ is non - decreasing and $G_{r} \geq G$

then by induction we get $G_{r}^{n} \geq G^{n}$ for all $\mathrm{n}$, so if $t \in(e \pi)^{-1} R, t^{\prime} \in(e \pi)^{-1} W$ and $>\quad t$, then $\frac{r}{s}=\lim _{n \rightarrow \infty} \frac{G_{r}^{n}(t)-t}{n}=\lim _{n \rightarrow \infty} \frac{G_{r}^{n}(t)}{n} \geq \lim _{n \rightarrow \infty} \frac{G_{r}^{n}(t \prime)}{n}=\frac{d}{m}$ then $\frac{r}{s} \geq \frac{d}{m}$..

Let $\mathrm{E}($.$) be the integer function.$

Theorem 1.2.9: Let $g: 8 \rightarrow 8$ be a continuous map of degree one. If $\frac{d}{m} \in L(g)$ such that $\frac{d}{m}$ is an end point of $L(g)$ then all periodic orbits of $g$ of period $m$ and rotation number $\frac{d}{m}$ are TPO.

Proof: Either $\frac{d}{m} \in Z$ such that $(d, m)=1$ then $\mathrm{m}=1$ then by proposition 1.2 .4 , g has a TPO. or $\frac{d}{m} \in Z$ let $\mathrm{F}$ be a lifting of $\mathrm{g}$ such that $\frac{d}{m}$ is the right end point of $L_{F}(f)=\left[a, \frac{d}{m}\right]$. let $G^{\prime}=G-E\left(\frac{d}{m}\right) \frac{d_{1}}{m}=\frac{d}{m}-E\left(\frac{d}{m}\right) \in L_{G}(g)$ then $(d, m)=1$ and $\frac{d_{1}}{m} \in(0,1)$ then by lemma 1.2.8, we get all the periodic orbits of period $\mathrm{m}$ and $\rho\left(F^{\prime}, z\right)=\frac{d^{\prime}}{m}$ are TPO. $\rho(G, z)=\rho\left(G^{\prime}, z\right)+E\left(\frac{d}{m}\right)=\frac{d_{1}}{m}+E\left(\frac{d}{m}\right)=\frac{d}{m}$. so T is TPO of period $\mathrm{m}$ and $p(G, z)=\frac{d}{m}$

\section{Refrences :}

[1] Alseda, L., Llibre, J., Misurewicz, M. and Simo, C. (1985) :" Twist periodic orbit and topological entropy for continuous maps of circle of degree one which have a fixed point, Ergod. Th. \& Dynam. Sys., 5,501-517.

[2] Block, L. Guckenheimer, J. Misurewicz, M. and Young, L.S. (1980): Periodic points and topological entropy of one dimensional maps. Springer Lect. Notes in Math., 819, 18-39.

[3] Misurewicz, M. (1982):Periodic points of maps of degree one of a circle ,Ergod. Th. \& Dynam., 2, 221-227.

[4] Misurewicz, M. (1984):Twist sets for maps of the circle" Ergod. Th.\& Dynam. Sys., 4, 391-404. 